\title{
CHARACTERISTIC CLASSES OF SURFACE BUNDLES
}

\author{
BY SHIGEYUKI MORITA
}

In this paper we define characteristic classes of surface bundles, namely smooth fibre bundles whose fibres are a closed orientable surface $\Sigma_{g}$ of genus $g \geq 2$, and announce some nontriviality results for them. As a consequence we obtain lower bounds for the Betti numbers of the mapping class group $M(g)$ of $\Sigma_{g}$.

It is known $[\mathbf{E E}]$ that the connected component of the identity of Diff $+\Sigma_{g}$, the group of orientation preserving diffeomorphisms of $\Sigma_{g}$, is contractible. Therefore $B$ Diff $_{+} \Sigma_{g}$ is a $K(M(g), 1)$. Now let $\xi$ be the tangent bundle along the fibres of an oriented surface bundle and let $e(\xi)$ be its Euler class. If we apply the Gysin homomorphsm to $e^{i+1}(\xi)$, we obtain an integral cohomology class of the base space of degree $2 i$. By naturality this defines certain cohomology classes $e_{i} \in H^{2 i}(M(g): \mathbf{Z})(i=1,2, \ldots) . M(g)$ acts on $H^{\mathbf{1}}\left(\Sigma_{g} ; \mathbf{Z}\right)$, preserving the symplectic form given by the cup product, so we obtain a homomorphism $M(g) \rightarrow \operatorname{Sp}(2 g ; \mathbf{Z})$, where $\operatorname{Sp}(2 g ; \mathbf{Z})$ is the group of all $2 g \times 2 g$ symplectic matrices with integral entries. This induces a homomorphism $M(g) \rightarrow \operatorname{Sp}(2 g ; \mathbf{R})$. Since $\operatorname{Sp}(2 g ; \mathbf{R})$ has $\mathrm{U}(g)$ as a maximal compact subgroup, we have a $g$-dimensional complex vector bundle $\eta$ on $K(M(g), 1)$. Let $c_{i}(\eta) \in H^{2 i}(M(g) ; \mathbf{Z})$ be its $i$ th Chern class. From the argument of Atiyah in $[\mathbf{A}]$ and the fact that $\eta$ is flat as a real vector bundle, we can conclude

$$
\begin{aligned}
& e_{2 i-1}=(-1)^{i}\left(2 i / B_{i}\right) s_{2 i-1}(c(\eta)) \quad(i=1,2, \ldots \text { and coefficients are in } \mathbf{Q}), \\
& s_{2 i}(c(\eta))=0
\end{aligned}
$$

where $s_{i}(c(\eta))$ stands for the characteristic class of $\eta$ corresponding to the formal sum $\sum_{j} t_{j}^{i}$, and $B_{i}$ is the $i$ th Bernoulli number. These two relations induce those among monomials of $e_{2 i-1}$ 's and the quotient

$$
\mathbf{Q}\left[e_{1}, e_{3}, \ldots\right] /(\text { relations })
$$

is naturally isomorphic to the relative Lie algebra cohomology $H^{*}(\mathfrak{s p}(2 g ; \mathbf{R})$, $\mathfrak{u}(g))$, which in turn is additively isomorphic to $H^{*}\left(S^{2} \times S^{4} \times \cdots \times S^{2 g} ; \mathbf{Q}\right.$ ) (see [BH]). It is known that $M(g)$ acts properly discontinuously on the Teichmüller space $T(g) \cong \mathbf{R}^{6 g-6}$ with noncompact quotient $\mathcal{M}_{g}$, the moduli space for Riemann surfaces of genus $g$. Hence $\operatorname{vcd}(M(g)) \leq 6 g-7$. Thus any monomial of $e_{i}$ 's of degree $\geq 6 g-6$ vanishes. To sum up we have a homomorphism

$$
\phi: \mathbf{Q}\left[e_{1}, e_{2}, \ldots\right] /(\text { above relations }) \rightarrow H^{*}(M(g) ; \mathbf{Q}) ;
$$

here we use the letters $e_{i}$ for both symbolic and actual meanings. Since $\operatorname{vcd}(M(g))$ is conjectured to be $3 g-3[\mathbf{H v}], \phi$ will surely still have a large kernel. Our main results are

Received by the editors July 26, 1983.

1980 Mathematics Subject Classification. Primary 55R40, 57N05; Secondary 32G15. 
THEOREM 1. For any $k \in \mathbf{N}$ there exists a natural number $g(k)$ such that the elements $e_{1}, \ldots, e_{k}$ are all nontrivial in $H^{*}(M(g) ; \mathbf{Q})$ if $g \geq g(k)$.

COROLLARY 2. The natural surjective homomorphism Diff $+\Sigma_{g} \rightarrow M(g)$ does not have a right inverse if $g \geq g(3)$ (we can take 86 for $g(3)$ ). In fact the induced homomorphism $H_{2 i}\left(\operatorname{Diff}_{+} \Sigma_{g}\right) \rightarrow H_{2 i}(M(g))$ is not surjective for $i=3,4, \ldots, k$ if $g \geq g(k)$ (here we consider $\operatorname{Diff}_{+} \Sigma_{g}$ as a discrete group).

This result should be compared with the recent affirmative solution of the Nielsen realization problem by Kerckhoff $[\mathbf{K e}]$.

THEOREM 3. For any $k \in \mathbf{N}$ there exists a natural number $g^{\prime}(k)$ such that the 2ith Betti number $b_{2 i}(M(g))$ of $M(g)$, which is equal to $b_{2 i}\left(\mathcal{M}_{g}\right)$, is at least $i$ for all $i=1, \ldots, k$ if $g \geq g^{\prime}(k)$.

We mention that Harer [Ha] has proved that $b_{2}(M(g))=1$ for $g \geq 5$. Since "half" of our characteristic classes come from $\operatorname{Sp}(2 g ; \mathbf{Z})$, we also have information on the homomorphism $H_{2 i}(M(g)) \rightarrow H_{2 i}(\operatorname{Sp}(2 g ; \mathbf{Z}))$. We omit the precise statement.

SKETCH OF PROOFS. The proofs of the above results are given by constructing sufficiently many surface bundles with nontrivial characteristic classes. Roughly speaking we apply the method of Atiyah $[\mathbf{A}]$ (see also [Ko]) iteratively. To be more precise, let $\pi: E \rightarrow X$ be a surface bundle with fibre $\Sigma_{g}$. We assume that $X$ is an iterated surface bundle. Given $\left(n, n^{\prime}\right) \in \mathbf{N} \times \mathbf{N}$, an " $\left(n, n^{\prime}\right)$-construction on $\pi: E \rightarrow X$ " is described by the following diagram of surface bundles:

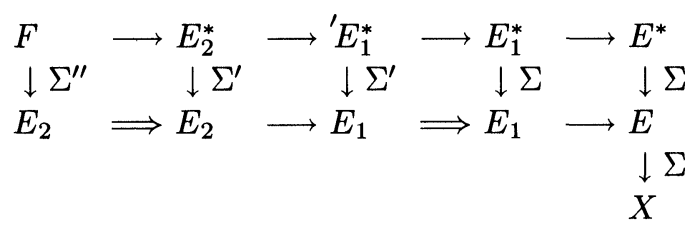

Here $E^{*} \rightarrow E$ is the pull back bundle $\pi^{*}(E)$. $E^{*}$ contains a cross-section $D$ as the diagonal. $E_{1} \rightarrow E$ is a covering map which kills first the action of $\pi_{1}(E)$ on $H^{1}$ (fibre; $\left.\mathbf{Z} / n n^{\prime}\right)$ and then kills $H^{1}\left(; \mathbf{Z} / n n^{\prime}\right) . E_{1}^{*} \rightarrow E^{*}$ is the pull back by this map. ' $E_{1}^{*} \rightarrow E_{1}^{*}$ is a fibrewise $n n^{\prime}$-fold covering map. $E_{2} \rightarrow E_{1}$ is a covering map which satisfies the condition: the homology class of the inverse image $D^{\prime}$ of $D$ under the map $E_{2}^{*} \rightarrow E^{*}$ is divisible by $n$. The assumption that $X$ is an iterated surface bundle guarantees the existence of such a covering. Finally $F \rightarrow E_{2}^{*}$ is an $n$-fold cyclic covering ramified along $D^{\prime} . \quad F \rightarrow E_{2}$ is a surface bundle with fibre $\Sigma^{\prime \prime}$ whose genus is $n^{2} n^{\prime} g-\frac{1}{2} n(n+1) n^{\prime}+1$. The $(2,1)$-construction on the trivial surface bundle $\Sigma_{g} \rightarrow$ pt is nothing but Atiyah's method in $[\mathbf{A}]$. Theorem 1 is proved by calculating $e_{k}$ of surface bundles which are defined by applying $\left(n_{j}, n_{j}^{\prime}\right)$-constructions on $\Sigma_{g} \rightarrow$ pt successively $(j=1, \ldots, k)$. It turns out that $e_{k}$ of such a surface bundle is $(g-1)$ times a nontrivial polynomial of $n_{j}, n_{j}^{\prime}$ 's. Since such surface bundles admit multivalued cross-sections, once the statement of Theorem 1 is proved for one $g_{0}$, it holds for all $g \geq g_{0}$. Corollary 2 follows from Theorem 1 and the Bott vanishing theorem $[\mathbf{B}]$. We can also compute characteristic classes other 
than $e_{k}$. It turns out that $e_{i_{1}}^{d_{1}} \cdots e_{i_{s}}^{d_{s}}\left(\sum_{j} i_{j} d_{j}=k\right)$ is a linear combination of $(g-1),(g-1)^{2}, \ldots,(g-1)^{d_{1}+\cdots+d_{s}}$ with coefficients in polynomials of $n_{j}, n_{j}^{\prime}$ 's. Theorem 3 follows from this.

It is very likely that these examples of surface bundles are enough to prove the injectivity of $\phi$ in small degrees. However, necessary computations for that are extremely complicated. Also it seems to be interesting to test the surjectivity of $\phi$ by examining these examples because it is by no means clear that characteristic numbers of a surface bundle which is obtained by applying an $\left(n, n^{\prime}\right)$-construction on another surface bundle depend only on those of the latter. The details together with these points will appear elsewhere.

\section{REFERENCES}

[A] M. F. Atiyah, The signature of fibre-bundles, Global Analysis, Papers in Honor of K. Kodaira, Tokyo Univ. Press, 1969, pp. 73-84.

[BH] A. Borel and F. Hirzebruch, Characteristic classes and homogeneous spaces. I, Amer. J. Math. 80 (1958), 459-538.

[B] R. Bott, On a topological obstruction to integrability, Global Analysis, Proc. Sympos. Pure Math., vol. 16, Amer. Math. Soc., Providence, R.I., 1970, pp. 127-131.

[EE] C. J. Earle and J. Eells, The diffeomorphism group of a compact Riemann surface, Bull. Amer. Math. Soc. 73 (1967), 557-559.

[Ha] J. Harer, The second homology group of the mapping class group of an orientable surface, Invent. Math. 72 (1983), 221-239.

[Hv] W. J. Harvey, Geometric structures of surface mapping class groups, Homological Group Theory, London Math. Soc., Lecture Notes No. 36, Cambridge Univ. Press, 1979, pp. 255269. 265 .

[Ke] S. P. Kerckhoff, The Nielsen realization problem, Ann. of Math. (2) 117 (1983), 235-

[Ko] K. Kodaira, A certain type of irregular algebraic surface, J. Analyse Math. 19 (1967), $207-215$.

DePARTMENT OF MATHEMATICS, UNIVERSITy OF TOKYO, TOKYO, JAPAN 